\title{
On the Elliptic Stark Conjecture at Primes of Multiplicative Reduction
}

\author{
Daniele CasazZa \& Victor Rotger
}

ABSTRACT. In [DLR], Darmon, Lauder, and Rotger formulated a $p$ adic elliptic Stark conjecture for the twist of an elliptic curve $E / \mathbb{Q}$ by the self-dual tensor product $\rho_{1} \otimes \rho_{2}$ of two odd and two-dimensional Artin representations. These authors provided abundant numerical evidence and proved the conjecture in the special setting where $p$ is a prime of good reduction for $E$ and $\rho_{1}$ and $\rho_{2}$ are induced from finite-order characters $\psi_{g}, \psi_{h}$ of the same imaginary quadratic field. The key step in their proof is a factorization of one-variable $p$-adic $L$-functions, where $\psi_{g}$ varies in a $p$-adic family of Hecke characters.

The main goal of this article is to prove a new case of the conjecture, placing ourselves in the setting where $p$ is a prime of multiplicative reduction for $E$. In order to achieve our theorem, we need to work with two-variable $p$-adic $L$-functions, where the weight 2 cusp form associated with $E$ also moves independently along a Hida family. Our main result then follows from a factorization of $p$-adic $L$-series extending to two variables the one obtained in [DLR]. On the way we also generalize to our setting the results obtained in [CR].

\section{CONTENTS}

1. Introduction

2. Garrett-Hida $p$-adic $L$-function

3. Castellàs Two-variable $p$-adic $L$-function

4. Katz $p$-adic $L$-function

5. Proof of Theorem 1.1

6. An Explicit Formula

Acknowledgements. 


\section{INTRODUCTION}

Fix a prime number $p \geq 3$ and fix three positive integers $N_{f}, N_{g}, N_{h}$ such that $p \nmid N_{f} N_{g} N_{h}$. Set $N=\operatorname{lcm}\left(N_{f}, N_{g}, N_{h}\right)$ and let $\chi:(\mathbb{Z} / N \mathbb{Z})^{\times} \rightarrow \mathbb{C}^{\times}$be a Dirichlet character. Let

$$
f \in S_{2}\left(p N_{f}\right), g \in M_{1}\left(N_{g}, \bar{\chi}\right), h \in M_{1}\left(N_{h}, \chi\right)
$$

be a triple of newforms of weights $(2,1,1)$, levels $\left(p N_{f}, N_{g}, N_{h}\right)$, and nebentype characters $(1, \bar{X}, \chi)$.

Assume for simplicity that the Fourier coefficients of $f$ are rational, and let $E / \mathbb{Q}$ be an elliptic curve in the isogeny class associated with $f$ by Eichler-Shimura. Let also $\varrho_{g}, \varrho_{h}$ denote the odd, two-dimensional Artin representations associated by Deligne-Serre with $g$ and $h$. This paper focuses on the arithmetic of the elliptic curve $E$ twisted by the four-dimensional Artin representation

$$
\varrho_{g h}:=\varrho_{g} \otimes \varrho_{h}
$$

which is self-dual because the nebentype characters of $g$ and $h$ are inverse to each other.

Let $L\left(E, \varrho_{g h}, s\right)$ denote the Hasse-Weil-Artin $L$-series associated with the compatible system of Galois representations afforded by the twist of $E$ by $\varrho_{g h}$. A direct consequence of the Eichler-Shimura and Deligne-Serre constructions is the identity

$$
L\left(E, \varrho_{g h}, s\right)=L(f, g, h, s),
$$

which recasts the $L$-function associated with the pair $\left(E, \varrho_{g h}\right)$ as Garrett's [Gar] triple-product $L$-function attached to the triple of eigenforms $(f, g, h)$.

Assuming that $\left(p N_{f}, N_{g} N_{h}\right)=1$, the global sign of the functional equation of $L\left(E, \varrho_{g h}, s\right)$ is +1 . We refer to, for example, [Pr90] for a proof of this simple fact and for similar assertions under more relaxed hypotheses.

Assume further that $L\left(E, \varrho_{g h}, s\right)$ vanishes at the central critical point $s=1$. It follows that the order of vanishing of $L\left(E, \varrho_{g h}, s\right)$ at $s=1$ is even, and hence at least 2.

In [DLR], Darmon, Lauder, and one of the authors of this note introduced certain $p$-adic iterated integrals attached to the triple $(f, g, h)$, which they view as $p$-adic avatars of the leading term of $L\left(E, \varrho_{g h}, s\right)$ at $s=1$. These iterated integrals are defined as linear forms

$$
\begin{aligned}
S_{2}(N p)_{L}[f] \times M_{1}(N p, \chi)_{L}^{\vee}\left[g_{\alpha}\right] \times M_{1}(N p, \chi)_{L}[h] & \longrightarrow \mathbb{C}_{p}, \\
(\breve{f}, \breve{\gamma}, \breve{h}) & \longmapsto \int_{\breve{\gamma}} \breve{f} \cdot \breve{h},
\end{aligned}
$$

on the space of triples of test vectors of level $N p$ on which the good Hecke operators act with the same system of eigenvalues as $f, g_{\alpha}$, and $h$, respectively. Here, 
$g_{\alpha}$ is a choice of (necessarily ordinary) $p$-stabilization of $g$, on which $U_{p}$ acts with eigenvalue $\alpha$.

Under our hypotheses and an additional assumption referred to as Hypothesis $\mathrm{C}$ in [DLR] on the local geometry of the points corresponding to the ordinary stabilisations of $g$ in the eigencurve, the authors of [DLR] propose a conjectural formula for these iterated integrals which may be regarded as a $p$-adic avatar of a putative Gross-Zagier formula in rank 2.

More precisely, these iterated integrals are expected to vanish systematically when the second derivative $L^{\prime \prime}\left(E, \varrho_{g h}, 1\right)$ vanishes; if instead $L^{\prime \prime}\left(E, \varrho_{g h}, 1\right) \neq 0$, the main conjecture of $[D L R]$ predicts that the values of the iterated integrals attached to $(f, g, h)$ encode the logarithm of a suitable Gross-Stark unit and a $2 \times$ $2 p$-adic regulator involving the formal group logarithms of certain Stark points in the Mordell-Weil group of $E$ over the number field cut out by $\varrho_{g h}$. This conjecture was christened in [DLR] as the elliptic Stark conjecture.

The main theorem of [DLR, Chapters 2,3] shows that the elliptic Stark conjecture holds when $p$ is a prime of good reduction for $E$, while $g$ and $h$ are binary theta series attached to a common imaginary quadratic field $K$ in which $p$ splits. This is done by recasting both sides of the conjectural formula, that is to say, $p$ adic iterated integrals on one side and elliptic units and Heegner points on the other, as values of various $p$-adic $L$-functions at points lying outside their region of interpolation.

The first main theorem of this article extends the above result to the case where $p$ is a prime of multiplicative reduction of $E$. In order to state the result precisely, let us briefly review the ingredients appearing in its formulation.

Fix throughout embeddings $\overline{\mathbb{Q}} \subset \mathbb{C}$ and $\overline{\mathbb{Q}} \subset \mathbb{C}_{p}$ so that every number field shall be regarded without further mention as a subfield of both $\mathbb{C}$ and $\mathbb{C}_{p}$.

Let $K$ be an imaginary quadratic field of discriminant $-D_{K}$, and let us denote by $\chi_{K}$ the quadratic Dirichlet character associated with the extension $K / \mathbb{Q}$. Assume that $p$ splits in $K$, that is, $\chi_{K}(p)=1$.

Let $g$ and $h$ be theta series that are associated with two finite-order characters $\psi_{g}, \psi_{h}: G_{K} \longrightarrow L^{\times}$of $K$ of conductors $\mathfrak{c}_{g}$ and $\mathfrak{c}_{h} \subseteq \mathcal{O}_{K}$, respectively, with values in a finite extension $L$ of $\mathbb{Q}$. They are weight-one forms of levels $D_{K} \mathrm{~N}_{K / \mathbb{Q}}\left(\mathfrak{c}_{\mathfrak{g}}\right)$ and $D_{K} \mathrm{~N}_{K / \mathbb{Q}}\left(\mathfrak{c}_{h}\right)$, having nebentype characters $\chi_{g}$ and $\chi_{h}$, respectively.

Define $\psi_{g}^{\prime}: G_{K} \longrightarrow L^{\times}$to be the $\operatorname{Gal}(K / \mathbb{Q})$-conjugate of the character $\psi_{g}$, namely, the character given by the rule $\psi_{g}^{\prime}(\sigma)=\psi_{g}\left(\tau \sigma \tau^{-1}\right)$ for any $\sigma \in G_{K}$ and any $\tau \in G_{\mathbb{Q}} \backslash G_{K}$.

We assume that $g$ satisfies the classicality hypotheses of [DLR] at the prime $p$. As explained in that paper, this is automatically satisfied when the following hold:

- $\psi_{g} / \psi_{g}^{\prime}=1$ (i.e., $g$ is an Eisenstein series).

- $\psi_{g} / \psi_{g}^{\prime} \neq 1$ (i.e., $g$ is cuspidal), if we impose $\psi(\wp) \neq \psi^{\prime}(\wp)$ and that $\rho_{g}$ is not induced from a character of a real quadratic field in which $p$ splits. 
As above, we assume that $\chi_{h}=\chi_{g}^{-1}(=\chi)$, which implies that the characters

$$
\psi_{1}=\psi_{g} \psi_{h} \quad \text { and } \quad \psi_{2}=\psi_{g} \psi_{h}^{\prime}
$$

are ring class characters of $K$, associated with orders $\mathcal{O}_{c_{1}}$ and $\mathcal{O}_{c_{2}}$ in $\mathcal{O}_{K}$ of conductors $c_{1}$ and $c_{2}$, respectively. Let $H$ denote the ring class field associated with the order $\mathcal{O}_{c}$ in $K$ of conductor $c:=\operatorname{lcm}\left(c_{1}, c_{2}\right)$ of $K$, which contains the fields cut out by $\psi_{1}$ and $\psi_{2}$.

A simple exercise shows that the Artin representation $\varrho_{g h}=\varrho_{g} \otimes \varrho_{h}$ decomposes as

$$
\varrho_{g h}=\rho_{1} \oplus \rho_{2}=V_{\psi_{1}} \oplus V_{\psi_{2}},
$$

and the Artin formalism in turn implies that there is a factorisation of $L$-series

$$
\begin{aligned}
L\left(E, \varrho_{g h}, s\right) & =L\left(E, \rho_{1}, s\right) \cdot L\left(E, \rho_{2}, s\right) \\
& =L\left(E / K, \psi_{1}, s\right) \cdot L\left(E / K, \psi_{2}, s\right) .
\end{aligned}
$$

Recall that

$$
N=\operatorname{lcm}\left(N_{f}, N_{g}, N_{h}\right)=\operatorname{lcm}\left(N_{f}, D_{K} \mathrm{~N}_{K / \mathbb{Q}}\left(\mathfrak{c}_{g}\right), D_{K} \mathrm{~N}_{K / \mathbb{Q}}\left(\mathfrak{c}_{h}\right)\right) .
$$

Assume in this introduction that $N$ is square-free, $\left(N_{f} D_{K}, c\right)=1$, and the following assumption holds (which is exactly the one imposed in Section 4.1 of [BDP13]):

Heegner hypothesis: All prime divisors of $N_{f}$ split or ramify in $K$, and the local signs at primes dividing $\operatorname{gcd}\left(N_{f}, D_{K}\right)$ of $L\left(E, \rho_{1}, s\right)$ and $L\left(E, \rho_{2}, s\right)$ are +1 .

We refer to [Gro, Sections 11 and 22] for more details about this hypothesis. As shown in [Gro], this assumption automatically implies that the local signs of $L\left(E, \rho_{1}, s\right)$ and $L\left(E, \rho_{2}, s\right)$ are +1 at all finite primes. Since the local sign at $\infty$ of $L\left(E, \rho_{i}, S\right)$ is -1 , this assumption implies that the global signs of the functional equations satisfied by $L\left(E / K, \psi_{1}, s\right)$ and $L\left(E / K, \psi_{2}, s\right)$ are -1 ; hence, the analytic ranks $r_{\text {an }}\left(E, \varrho_{\psi_{1}}\right)$ and $r_{\text {an }}\left(E, \varrho_{\psi_{2}}\right)$ are both odd.

It also follows from $(1.1)$ that $L\left(E, \varrho_{g h}, 1\right)=0$, and thus Hypothesis $B$ of [DLR] is satisfied. Moreover, since the local sign at any place of $L\left(E, \varrho_{g h}, s\right)$ is the product of the local signs of $L\left(E / K, \psi_{1}, s\right)$ and of $L\left(E / K, \psi_{2}, s\right)$, it follows that Hypothesis A in [DLR] holds.

Finally, the Heegner hypothesis also implies there exists an integral ideal $\mathfrak{N}$ in $\mathcal{O}_{K}$ such that $\mathrm{N}_{K / \mathbb{Q}}(\mathfrak{N})=N_{f}$. We fix such an ideal for the remainder of the article.

Let $X$ denote the modular curve associated in [LRdV, Theorem A] with the triple $(E, K, c)$, and let $\pi_{E}: X \rightarrow E$ be a modular parametrization of $E$.

Under our assumptions, we let $X(H)_{c} \subset X(H)$ denote the set of Heegner points attached to the order $\mathcal{O}_{c}$. After fixing a choice of $P \in X(H)_{c}$, any ring class 
character $\psi$ of conductor $c$ gives rise to a degree zero $H$-rational divisor on $X$ by setting

$$
D_{\psi}:= \begin{cases}\sum_{\sigma \in G} \psi^{-1}(\sigma) P^{\sigma}, & \text { if } \psi \neq 1, \\ \sum_{\sigma \in G} P^{\sigma}-\# G \cdot \infty & \text { if } \psi=1,\end{cases}
$$

where $\infty$ is the cusp at infinity and $G:=\mathrm{Gal}(H / K)$. The Heegner point attached to $\psi$ is then defined to be

$$
P_{\psi}:=\pi_{E}\left(D_{\psi}\right) \in E(H) \otimes L .
$$

Set $\psi_{0}:=\psi_{g} / \psi_{g}^{\prime}$, and note that this is again a ring class character of $K$. Fix an elliptic unit $u$ in the field $H_{g}$ cut out by $\psi_{0}$ as in [DLR, Section 3.1], and define

$$
u_{\psi_{0}}=\sum_{\sigma \in G} \psi_{0}^{-1}(\sigma) u^{\sigma} \in\left(\mathcal{O}_{H_{g}}^{\times}\right) \otimes L .
$$

Let $\omega_{E}$ denote the Néron differential 1-form of $E$. The pull-back of this form to $X$ is a non-zero rational multiple of $\omega_{f}$, the canonical differential 1 -form on $X$ associated with $f$. Hence, $\pi_{E}^{*}\left(\omega_{E}\right)=c_{E} \cdot \omega_{f}$ for some $c_{E} \in \mathbb{Q}^{\times}$, usually referred to as the Manin constant of the modular parametrization. We refer to [ARS] for a survey of known results about the Manin constant.

Fix embeddings of $H_{g} \subseteq H$ into $\overline{\mathbb{Q}}_{p} \subset \mathbb{C}_{p}$, and let $H_{p}$ denote the closure of $H$ in $\mathbb{C}_{p}$. Let

$$
\log _{E, p}: E\left(H_{p}\right) \longrightarrow H_{p}
$$

denote the formal group logarithm associated with $\omega_{E}$. Let also

$$
\log _{p}: \mathcal{O}_{H_{p}}^{\times} \longrightarrow \mathcal{O}_{H_{p}}
$$

denote the usual $p$-adic logarithm. The main theorem of this note is the following.

Theorem 1.1. If either $L^{\prime}\left(E / K, \psi_{1}, 1\right)$ or $L^{\prime}\left(E / K, \psi_{2}, 1\right)$ vanishes, the iterated integrals attached to the triple $(f, g, h)$ vanish identically.

Otherwise, there exists a finite extension $L$ of $\mathbb{Q}\left(\psi_{g}, \psi_{h}\right)$, a scalar $\lambda \in L^{\times}$, and test vectors

$$
\left(\breve{f}, \breve{\gamma}_{g_{\alpha}}, \breve{h}\right) \in S_{2}(N p)_{L}[f] \times M_{1}(N p, \chi)_{L}^{\vee}\left[g_{\alpha}\right] \times M_{1}(N p, \chi)_{L}[h]
$$

for which

$$
\int_{\breve{\gamma}_{g_{\alpha}}} \breve{f} \cdot \breve{h}=\lambda \cdot \frac{\log _{E, p}\left(P_{\psi_{1}}\right) \log _{E, p}\left(P_{\psi_{2}}\right)}{\log _{p}\left(u_{\psi_{0}}\right)} .
$$


The second main result of this note is a precise formula for the value of $\lambda$ appearing in the above statement, in a particular setting.

Theorem 1.2. Assume $N_{f}=D_{K}$ and $g=h=\operatorname{Eis}\left(1, \chi_{K}\right)$ are the Eisenstein series associated with the pair of Dirichlet characters $\left(1, \chi_{K}\right)$. Let $h_{K}=\left|\operatorname{Pic}\left(\mathcal{O}_{K}\right)\right|$ and $g_{K}=\left|\operatorname{Pic}\left(\mathcal{O}_{K}\right) / \operatorname{Pic}^{2}\left(\mathcal{O}_{K}\right)\right|$ denote the class number and genus number of $K$. Then,

$$
\lambda=\frac{\left(p-a_{p}(f) \psi(\wp)\right)^{2}}{p \cdot c_{E}^{2}} \cdot \frac{\lambda_{0}}{h_{K} g_{K}},
$$

where

$$
\lambda_{0}=\left\{\begin{array}{l}
\frac{1}{p-1} \\
\frac{12}{p-(p+1) \psi^{2}(\bar{\wp})+\psi^{4}(\bar{\wp})}
\end{array}\right.
$$

if $\psi^{2}=1$, that is to say, if $g$ is an Eisenstein series, if $\psi^{2} \neq 1$, that is to say, if $g$ is cuspidal.

The proof of these results is not a straightforward generalization of [DLR] and [CR] respectively, mainly because of the following reasons:

(I) One of the main ingredients in the proofs of Theorems 1.1 and 1.2 is the main result of Bertolini, Darmon, and Prasanna in [BDP13], which is proved therein under the assumption that $p$ is a prime of good (although not necessarily ordinary) reduction.

(II) Another crucial ingredient in our proofs is the use of various $p$-adic $L$ functions, like Garrett-Hida's $p$-adic $L$-function constructed in [DR1], [DLR, Chspter 2], and Bertolini-Darmon-Prasanna's $p$-adic $L$-function described in [BDP13, Section 5]. In the interpolation formulae relating these functions to critical values of classical $L$-series there appear certain auxiliary constants as, for instance, the ones denoted $C_{v}$ in Proposition 2.1 of [DLR], and also the one denoted $\omega(f, \psi)$ in [BDP13, (5.1.11)]. When $p$ is a prime of bad reduction, it is a rather subtle problem to analyze the $p$-adic valuation of these constants and its variation along Hida families of modular forms.

(III) To find an explicit formula for the scalar $\lambda$ appearing in Theorem 3.3 of [DLR], it was crucial in [CR] to assume that the levels of the three modular forms $f, g$, and $h$ were all equal, as otherwise the computations became too daunting. In the setting under study here, this assumption is not feasible because $p$ divides the level of $f$ but not the levels of $g$ and $h$.

In order to overcome these difficulties, we add an extra $p$-adic variable to the computations of [DLR, Chapter 2] by allowing the modular form $f$ to vary in a Hida family. While in [DLR] $f$ was fixed throughout the article, here we adopt the strategy of regarding it as a member of a Hida family $\mathbf{f}$ because the higherweight specialisations $\mathbf{f}_{k}, k>2$, are (as opposite to $f$ itself) old at $p$. This allows us to work essentially in a setting where $p$ is a prime that behaves "as if it does 
not divide the level," and thus the necessary computations can then be performed more easily.

In the process of our proof, we prove in Theorem 6.1 a factorisation formula of two-variable $p$-adic $L$-functions involving Castellà's recent generalisation of [BDP13] that the reader may find of independent interest. Just as the analogous one-variable formula proved in [DLR, Theorem 3.9] has found recently striking applications to the arithmetic of elliptic curves, we expect that our twovariable formula shall be also useful in this direction (cf. the forthcoming work of Bertolini and Darmon on Perrin-Riou's conjecture [PR93] for elliptic curves and Castellàs work in progress on the Iwasawa theory of elliptic curves over imaginary quadratic fields).

\section{GARRETT-HIDA $p$-ADIC $L$-FUNCTION}

The starting point in the proof of Theorem 1.1 is the interpolation formula satisfied by the Garrett-Hida $p$-adic $L$-function associated with a triple of Hida families passing through $f, g$, and $h$, which we now recall.

Fix a rational prime $p \geq 3$, set $\Gamma=1+p \mathbb{Z}_{p}$, and denote by $\omega: \mathbb{Z} / p \mathbb{Z} \rightarrow \mu_{p-1}$ the usual Teichmuller character modulo $p$.

Let $\Lambda=\mathbb{Z}_{p} \llbracket \Gamma \rrbracket$ denote the Iwasawa algebra associated with $\Gamma$. Define the formal scheme of weights as $\chi=\chi_{\Lambda}:=\operatorname{Spf}(\Lambda)$. For any complete $\mathbb{Z}_{p}$-algebra $R$, the set of $R$-valued points of $\mathcal{X}$ may be identified with

$$
\chi(R)=\operatorname{Hom}_{\text {cont }}(\Lambda, R) \simeq \operatorname{Hom}\left(\Gamma, R^{\times}\right) .
$$

As usual, the set $\mathbb{Z}_{\geq 2}$ can be embedded in $\chi\left(\mathbb{Z}_{p}\right)$ by identifying an integer $k$ with the character $v_{k}: \Gamma \longrightarrow \mathbb{Z}_{p}^{\times}$sending $z$ to $z^{k}$. We let $\mathcal{X}^{\mathrm{cl}}$ denote the set of such classical points.

Given a finite flat extension $\tilde{\Lambda}$ of $\Lambda$, and defining $\tilde{X}:=\chi_{\tilde{\Lambda}}$, there is a natural projection $\tilde{X} \rightarrow \mathcal{X}$ induced by the inclusion $\Lambda \hookrightarrow \tilde{\Lambda}$. We define $\tilde{X}^{\mathrm{cl}}$ as the pullback of $\mathcal{X}^{\mathrm{cl}}$ under this map. The set $\bar{X}^{\mathrm{cl}}$ is called the set of arithmetic primes of $\bar{\Lambda}$. By definition, any $v \in \tilde{X}^{\mathrm{cl}}$ lies above $v_{k} \in \chi^{\mathrm{cl}}$ for some $k=k(v) \geq 2$, which we refer to as the weight of $v$.

Definition 2.1. Let $N_{f} \geq 1$ be an arbitrary integer not divisible by $p$, and $\chi$ be a Dirichlet character modulo $N_{f}$. A Hida family of tame level $N_{f}$ and tame character $\chi$ is a quadruple $\left(\Lambda_{f}, \mathcal{U}_{f}, \mathcal{U}_{f}^{\mathrm{cl}}, \mathbf{f}\right)$ where the following hold:

- $\Lambda_{f}$ is a finite flat extension of $\Lambda$.

- $\mathcal{U}_{f} \subset \chi_{f}:=\chi_{\Lambda_{f}}$ is a rigid-analytic open subvariety.

- $\mathcal{U}_{f}^{\mathrm{cl}} \subset \mathcal{U}_{f}\left(\mathbb{C}_{p}\right)$ is a dense subset for the rigid analytic topology.

- $\mathbf{f}=\sum \mathbf{a}_{n} q^{n} \in \Lambda_{f} \llbracket q \rrbracket$ is a formal $q$-series such that for all $v \in \mathcal{U}_{f}^{\mathrm{cl}}$ of weight $k=k(v)$,

$$
v(\mathbf{f})=\sum_{n \geq 1} v\left(\mathbf{a}_{n}\right) q^{n} \in S_{k}\left(p N_{f}, \chi \omega^{k_{0}-k}\right)
$$


is the $q$-expansion of an ordinary eigenform of weight $k$, level $p N_{f}$, and nebentype $x \omega^{k_{0}-k}$, for some $k_{0} \in\{0, \ldots, p-2\}$.

Remark 2.2. In the literature one often allows a richer plethora of classical specializations, including twists by characters of $p$-power conductor of $\Gamma$. For our purposes it suffices to restrict our focus to the case in which this character is trivial. Given an arithmetic prime $v \in \mathcal{U}_{f}^{\mathrm{cl}}$ over the classical point $v_{k} \in \mathcal{X}$, it will often be harmless to abuse notation and write $\mathbf{f}_{k}$ instead of $v(\mathbf{f})$.

Remark 2.3. To simplify further the notation, we shall restrict the classical weights to those $k \in \mathbb{Z}_{\geq 1}$ for which $k \equiv k_{0}(\bmod p-1)$, so that the nebentype character of $\mathbf{f}_{k}$ will be $\chi$. Under this condition, the specialisation $\mathbf{f}_{k}$ of the Hida family $\mathbf{f}$ at a point of weight $k>2$ is a classical eigenform which is always old at $p$ (cf. e.g. [Ho07, Lemma 2.1.5]), and there exists an eigenform $f_{k}$ of level $N$ such that $\mathbf{f}_{k}(q)=f_{k}(q)-\beta_{f_{k}} f_{k}\left(q^{p}\right)$ is the ordinary $p$-stabilization of $f_{k}$. Here, $\beta_{f_{k}}$ is the single non-unit root of the Hecke polynomial $T^{2}-a_{p}\left(f_{k}\right) T+\chi(p) p^{k-1}$. The Hecke operator $U_{p}$ acts on $\mathbf{f}_{k}$ with eigenvalue $\alpha_{f_{k}}$, the single $p$-adic unit root of the above polynomial.

If $k=2$, then $\mathbf{f}_{2}$ is a classical eigenform that may either be old or new $p$. In the former case, there exists as above an eigenform $f_{2}$ of level $N$ such that $\mathbf{f}_{2}$ is the ordinary $p$-stabilisation of $f_{2}$. In the latter case, we simply set $f_{2}=\mathbf{f}_{2}$ and $\alpha_{f_{2}}=a_{p}\left(f_{2}\right), \beta_{f_{2}}=0$.

If $k=1$, the $p$-adic modular form $\mathbf{f}_{1}$ may be classical or not: see [DG] for a discussion of this phenomenon.

Recall now the three classical eigenforms

$$
f \in S_{2}\left(p N_{f}\right), \quad g \in M_{1}\left(N_{g}, \bar{x}\right), \quad h \in M_{1}\left(N_{h}, \chi\right)
$$

introduced at the beginning of this note. Choose eigenforms

$$
\breve{f} \in S_{2}(N p)[f], \quad \breve{g} \in M_{1}(N, \bar{x})[g], \quad \breve{h} \in M_{1}(N, \chi)_{L}[h]
$$

with respect to the good Hecke operators, having the same eigenvalues as $f, g$, and $h$ respectively.

Since $g$ and $h$ have weight 1 , the roots of the Hecke polynomials

$$
\begin{aligned}
& T^{2}-a_{p}(g) T+\bar{\chi}(p)=\left(T-\alpha_{g}\right)\left(T-\beta_{g}\right), \\
& T^{2}-a_{p}(h) T+\chi(p)=\left(T-\alpha_{h}\right)\left(T-\beta_{h}\right)
\end{aligned}
$$

are all roots of unity. Fix arbitrary orderings $\left(\alpha_{g}, \beta_{g}\right),\left(\alpha_{h}, \beta_{h}\right)$ of these roots, and set

$$
\begin{array}{ll}
g_{\alpha}(q):=g(q)-\beta_{g} g\left(q^{p}\right), & h_{\alpha}(q):=h(q)-\beta_{h} h\left(q^{p}\right), \\
\breve{g}_{\alpha}(q):=\breve{g}(q)-\beta_{g} \breve{g}\left(q^{p}\right), & \breve{h}_{\alpha}(q):=\breve{h}(q)-\beta_{h} \breve{h}\left(q^{p}\right) .
\end{array}
$$


Theorems of Hida [Hi86] and Wiles [Wi88] ensure there exist Hida families $\breve{\mathbf{f}}, \breve{\mathbf{g}}, \breve{\mathbf{h}}$ of tame level $N$ such that, for suitable arithmetic primes of weights 2,1 , and 1 , we have

$$
\breve{\mathbf{f}}_{2}(q)=\breve{f}(q), \quad \breve{\mathbf{g}}_{1}(q)=\breve{g}_{\alpha}(q), \quad \breve{\mathbf{h}}_{1}(q)=\breve{h}_{\alpha}(q) .
$$

Define a dense subset of $\mathcal{U}_{f} \times \mathcal{U}_{g} \times \mathcal{U}_{h}$ as follows:

$$
\mathcal{U}_{f g h}^{\mathrm{cl}}:=\left\{(k, \ell, m) \in \mathcal{U}_{f}^{\mathrm{cl}} \times \mathcal{U}_{g}^{\mathrm{cl}} \times \mathcal{U}_{h}^{\mathrm{cl}} \mid \ell \geq k+m\right\} .
$$

Note that in [DR1], the authors constructed a three-variable $p$-adic $L$-function on $\mathcal{U}_{f} \times \mathcal{U}_{g} \times \mathcal{U}_{h}$ which is determined by an interpolation formula relating the values at points in $\mathcal{U}_{f g h}^{\mathrm{cl}}$ to central critical values of classical $L$-series: we refer to Proposition 2.3 of [DLR] for full-fledged details.

For our purposes here, it suffices to restrict the above $p$-adic $L$-function to $\mathcal{U}_{f g}:=\mathcal{U}_{f} \times \mathcal{U}_{g}$, regarded as a rigid-analytic sub-variety of $\mathcal{U}_{f} \times \mathcal{U}_{g} \times \mathcal{U}_{h}$, by setting the third variable to be the fixed point of weight one giving rise to $\breve{h}_{\alpha}$. Set also $\mathcal{U}_{f g}^{\mathrm{cl}}:=\left\{(k, \ell) \in \mathcal{U}_{f}^{\mathrm{cl}} \times \mathcal{U}_{g}^{\mathrm{cl}}, \ell \geq k+1\right\}$.

With these notation, the constructions of [DR1] and [DLR, Chapter 2] give rise to a two-variable $p$-adic $L$-function

$$
\mathcal{L}_{p}^{g}(\breve{\mathbf{f}}, \breve{\mathbf{g}}, \breve{h}): \mathcal{U}_{f g} \longrightarrow \mathbb{C}_{p}
$$

satisfying, for all $(k, \ell) \in \mathcal{U}_{f \mathfrak{g}}^{\mathrm{cl}}$, the interpolation property

$$
\begin{aligned}
& \mathcal{L}_{p}^{\mathcal{g}}(\breve{\mathbf{f}}, \breve{\mathbf{g}}, \breve{h}) \cdot \mathcal{L}_{p}^{g}\left(\breve{\mathbf{f}}^{*}, \breve{\mathbf{g}}^{*}, \breve{h}^{*}\right)(k, \ell) \\
&=\mathfrak{e}_{\mathrm{GH}}(k, \ell)^{2} \cdot \mathfrak{a}_{\mathrm{GH}}(k, \ell) \cdot \mathfrak{f}_{\mathrm{GH}}(k, \ell) \\
& \quad \times \prod_{v \mid N \infty} C_{v}\left(\breve{f}_{k}, \breve{g}_{\ell}, \breve{h}\right) \cdot \frac{L\left(f_{k} \otimes g_{\ell} \otimes h,(k+\ell-1) / 2\right)}{\left\langle g_{\ell}^{*}, g_{\ell}^{*}\right\rangle^{2}}
\end{aligned}
$$

where the following hold:

- For any modular form or Hida family $\varphi$ of character $\chi$, we set $\varphi^{*}=$ $\varphi \otimes \bar{\chi}$.

- $L\left(f_{k}, \otimes g_{\ell} \otimes h, s\right)$ is Garrett's triple-product complex $L$-function.

- $\mathfrak{a}_{\mathrm{GH}}(k, \ell)=((\ell+k-3) / 2) !^{2}((\ell-k-1) / 2) !^{2} \cdot \pi^{-2 \ell}$.

- $\mathfrak{e}_{\mathrm{GH}}(k, \ell)=\mathcal{E}\left(g_{\ell}, f_{k}, h\right) / \mathcal{E}_{0}\left(g_{\ell}\right) \mathcal{E}_{1}\left(g_{\ell}\right)=\mathcal{E}\left(g_{\ell}^{*}, f_{k}^{*}, h^{*}\right) / \mathcal{E}_{0}\left(g_{\ell}^{*}\right) \mathcal{E}_{1}\left(g_{\ell}^{*}\right)$ is the Euler factor introduced in [DR1, Theorem 1.3].

- $\mathfrak{f}_{\mathrm{GH}}(k, \ell)=A \cdot 2^{B}$ where $A=A(N) \in \mathbb{Q}^{\times}$and $B=B(k, \ell)$ is linear in $k, \ell$.

- $C_{v}\left(\breve{f}_{k}, \breve{g}_{\ell}, \breve{h}\right)$ is a constant in the field generated by the Fourier coefficients of $\breve{f}_{k}, \breve{g}_{\ell}$ and $\breve{h}$; thanks to the work of Watson and Woodbury 
[Wo1], when the three levels of the modular forms are square-free there exists a choice of test vectors $\left(\breve{f}_{k}, \breve{g}_{\ell}, \breve{h}\right)$ such that the constants

$$
W_{v}:=C_{v}\left(\breve{f}_{k}, \breve{g}_{\ell}, \breve{h}\right)
$$

satisfy that $W_{v}$ lie in $\mathbb{Q}^{\times}$for all $v \mid N$ and only depends on $N$ and $v$, but not on the weights $(k, \ell)$ (see [DLR, Remarks 2.2 and 2.4]).

When we evaluate this function at the point $(2,1) \in \mathcal{U}_{f \mathfrak{g}}$, which lies outside the region of classical interpolation, [DLR, Proposition 2.6] asserts that

$$
\mathcal{L}_{p}^{g}(\breve{\mathbf{f}}, \breve{\mathbf{g}}, \breve{h})(2,1)=\int_{\breve{\gamma}_{\alpha}} \breve{f}_{2} \cdot \breve{h}
$$

for a suitable choice of linear functional $\breve{\gamma}_{\alpha}: S_{1}(N p, \chi)_{L}\left[\breve{g}_{\alpha}^{*}\right] \rightarrow L$, with $L=$ $\mathbb{Q}\left(f_{2}, g_{1}, h\right)$.

\section{CASTELLÀ'S TWO-VARIABLE $p$-ADIC $L$-FUNCTION}

Consider the Hida family $\mathbf{f}$ as in the previous section. For any weight $\kappa \in \mathcal{U}_{f}^{\mathrm{cl}}$ such that $\kappa \equiv 2(\bmod p-1)$, let $f_{\kappa}$ denote the modular form of weight $\kappa$ whose $p$-stabilization equals the weight $\kappa$ specialization of $\mathbf{f}$, as in Remark 2.3.

Let $K=\mathbb{Q}\left(\sqrt{-D_{K}}\right), D_{K} \geq 7$, be an imaginary quadratic field. We keep the same assumptions as in the Introduction, so in particular we assume the Heegner hypothesis and we let $p=\wp \bar{\gamma}$ be an odd prime that splits in $K$.

A Hecke character $\psi$ of $K$ of infinity type $\left(\kappa_{1}, \kappa_{2}\right) \in \mathbb{Z} \times \mathbb{Z}$ is a continuous homomorphism $\psi: \mathbb{A}_{K}^{\times} \longrightarrow \mathbb{C}^{\times}$satisfying

$$
\psi\left(\alpha \cdot x \cdot z_{\infty}\right)=\psi(x) z_{\infty}^{\kappa_{1}} \bar{z}_{\infty}^{\kappa_{2}}
$$

for every $\alpha \in K^{\times}, x \in \mathbb{A}_{K}^{\times}$, and $z_{\infty} \in K_{\infty}^{\times}=\mathbb{C}^{\times}$. With this convention, the Hecke character $\mathbf{N}_{K}$ induced by the norm from $K$ to $\mathbb{Q}$ has infinity type $(1,1)$. A Hecke character $\psi$ of infinity type $\left(\kappa_{1}, \kappa_{2}\right)$ is called anticyclotomic if its central character satisfies $\psi_{\mid \mathbb{A}_{\mathbb{Q}}^{\times}}=\mathbf{N}_{K}^{\kappa_{1}+\kappa_{2}}$.

Let $\mathfrak{c} \subseteq \mathcal{O}_{K}$ be an integral ideal, and set $N=\operatorname{lcm}\left(N_{f}, D_{K} \mathrm{~N}_{K / \mathbb{Q}}(\mathfrak{c})\right)$. Let $\Sigma_{\mathrm{cc}}$ denote the set of anticyclotomic Hecke characters of $K$ of conductor dividing $N$. For any Hecke character $\psi \in \Sigma_{\text {cc }}$ of infinity type $\left(\kappa_{1}, \kappa_{2}\right)$ such that we have $\kappa=\kappa_{1}+\kappa_{2} \in \mathcal{U}_{f}^{\mathrm{cl}}$, let $L\left(f_{\kappa}, \psi, s\right)$ denote the $L$-function associated with the twist of $f_{K}$ by $\psi$, that is, the $L$-function attached to the compatible system of Galois representations afforded by the tensor product $\varrho_{f_{K} \mid G_{K}} \otimes \psi$ of the (restriction to $G_{K}$ of) the Galois representations attached to $f_{\kappa}$ and the character $\psi$.

As usual, $L\left(f_{\kappa}, \psi, s\right)=\prod_{q} L^{(q)}\left(q^{-s}\right)$ is defined as a product of Euler factors ranging over the set of prime numbers. The Euler factors at the primes $q$ such that $q \nmid N$ are the same as that of the Rankin $L$-series $L\left(\theta_{\psi} \otimes f_{K}, S\right)$, but may differ 
at the primes $q$ such that $q \mid N$. Since $\psi$ is anticyclotomic, $s=0$ is the central critical point of the functional equation satisfied by $L\left(f_{\kappa}, \psi^{-1}, s\right)$.

For a given $\kappa \in \mathcal{U}_{f}^{\mathrm{cl}}$, let $\Sigma_{\kappa, \mathrm{cc}} \subset \Sigma_{\mathrm{cc}}$ be the subset of anticyclotomic Hecke characters in $\Sigma_{\text {cc }}$ of infinity type $\left(\kappa_{1}, \kappa_{2}\right)$ with $\kappa_{1}+\kappa_{2}=\kappa$. This set is naturally the disjoint union of the three subsets

$$
\begin{aligned}
& \Sigma_{\kappa, \mathrm{cc}}^{(1)}=\left\{\psi \in \Sigma_{\kappa, \mathrm{cc}} \text { of infinity type }(1,1)\right\}, \\
& \Sigma_{\kappa, \mathrm{cc}}^{(2)}=\left\{\psi \in \Sigma_{\kappa, \mathrm{cc}} \text { of infinity type }(\kappa+\lambda,-\lambda), \lambda \in \mathbb{Z}_{\geq 0}\right\}, \\
& \sum_{\kappa, \mathrm{cc}}^{\left(2^{\prime}\right)}=\left\{\psi \in \Sigma_{\kappa, \mathrm{cc}} \text { of infinity type }(-\lambda, \lambda+\kappa), \lambda \in \mathbb{Z}_{\geq 0}\right\} .
\end{aligned}
$$

Each of the three sets $\Sigma_{\kappa, \mathrm{cc}}^{(1)}, \Sigma_{\kappa, \mathrm{cc}}^{(2)}$, and $\Sigma_{\kappa, \mathrm{cc}}^{\left(2^{\prime}\right)}$ is dense in the completion $\hat{\Sigma}_{\kappa, \mathrm{cc}}$ of $\Sigma_{\kappa, \mathrm{cc}}$ with respect to the $p$-adic compact open topology as explained in [BDP13, Section 5.2]. Therein, those authors construct a $p$-adic $L$-function on $\hat{\Sigma}_{\kappa, \text { cc }}$, denoted $\mathcal{L}_{p}\left(f_{K} / K\right)$, interpolating the algebraic parts of the central critical values of $L\left(f_{\kappa}, \psi^{-1}, s\right)$ at characters $\psi$ in $\Sigma_{\kappa, \mathrm{cc}}^{(2)}$.

In [Ca1, Section 1], Castellà constructs a two-variable $p$-adic $L$-function $\mathcal{L}_{p}(\mathbf{f} / K)(\kappa, \psi)$ on the completion $\hat{\Sigma}_{\mathrm{cc}}$ of $\Sigma_{\mathrm{cc}}$, lifting the one-variable $p$-adic $L$ function of Bertolini-Darmon-Prasanna. To state this more precisely, note that $\hat{\Sigma}_{\mathrm{cc}}$ is naturally fibered over $\mathcal{U}_{f}$ and the fiber at a given point $\kappa \in \mathcal{U}_{f}^{\mathrm{cl}}$ is $\hat{\Sigma}_{\kappa, \mathrm{cc}}$. Then, for any $\kappa \in \mathcal{U}_{f}^{\mathrm{cl}}$, we have

$$
\mathcal{L}_{p}(\mathbf{f} / K)(\kappa,-)^{2}=\mathcal{L}_{p}\left(f_{K} / K\right) .
$$

Castellàs $p$-adic $L$-function is characterized by the following interpolation property: let $\kappa \in \mathcal{U}_{f}$ and let $\psi \in \Sigma_{\kappa, \mathrm{cc}}^{(2)}$ be a character of infinity type $(\kappa+\lambda,-\lambda)$. Then, [Ca1, Theorem 1.4] asserts that

$$
\begin{gathered}
\mathcal{L}_{p}(\mathbf{f} / K)(\kappa, \psi)^{2}=\mathfrak{a}_{\mathrm{BDP}}(\psi) \cdot \mathfrak{e}_{\mathrm{BDP}}(\kappa, \psi)^{2} \cdot \mathfrak{f}_{\mathrm{BDP}}(\kappa, \psi) \\
\times \frac{\Omega_{p}^{2 \kappa+4 \lambda}}{\Omega^{2 \kappa+4 \lambda}} \cdot L\left(f_{\kappa}, \psi^{-1}, 0\right),
\end{gathered}
$$

where

- $\mathfrak{a}_{\mathrm{BDP}}(\psi)=\lambda !(\kappa+\lambda-1) ! \pi^{\kappa+2 \lambda-1}$,

- $\mathfrak{e}_{\mathrm{BDP}}(\kappa, \psi)=\left(1-\alpha_{f_{\kappa}} \psi^{-1}(\bar{\wp})\right)\left(1-\beta_{f_{\kappa}} \psi^{-1}(\bar{\wp})\right)$,

- $\mathfrak{f}_{\mathrm{BDP}}(\kappa, \psi)=\left(2 /\left(c \sqrt{D_{K}}\right)\right)^{\kappa+2 \lambda-1} \cdot 2^{\sharp\left\{q \mid\left(D_{K}, N_{E}\right)\right\}} \cdot \omega\left(f_{\kappa}, \psi\right)^{-1}$,

with $\omega\left(f_{\kappa}, \psi\right)$ as defined in [BDP13, (5.1.11)].

If $\psi$ is a finite-order anticyclotomic character of conductor $c \mid \mathfrak{c}$, then $\psi^{-1} \mathbf{N}_{K}$ is a character of infinity type $(1,1)$, and hence lies outside the region of interpolation. The main theorems of [BDP13] and [Ca2, Theorem 2.10] assert that, for such $\psi$, the following formula holds:

$$
\mathcal{L}_{p}(\mathbf{f} / K)\left(2, \psi \mathbf{N}_{K}\right)= \pm \mathfrak{f}_{p}(f, \psi) \cdot \log _{\omega_{f}}\left(D_{\psi}\right),
$$


where $\mathfrak{f}_{p}(f, \psi)=\left(1-\alpha_{f_{2}} \psi^{-1}(\bar{\wp}) p^{-1}\right)\left(1-\beta_{f_{2}} \psi^{-1}(\bar{\wp}) p^{-1}\right)$, and $D_{\psi}$ is the class in $\operatorname{Jac}(X)$ of the divisor introduced in (1.2).

Since $\pi_{E}^{*}\left(\omega_{E}\right)=c_{E} \cdot \omega_{f}$ and $P_{\psi}=\pi_{E}\left(D_{\psi}\right)$, it follows that

$$
\log _{\omega_{f}}\left(D_{\psi}\right)=\frac{1}{c_{E}} \log _{\pi_{E}^{*}\left(\omega_{E}\right)}\left(D_{\psi}\right)=\frac{1}{c_{E}} \log _{E, p}\left(P_{\psi}\right),
$$

and hence

$$
\mathcal{L}_{p}(\mathbf{f} / K)\left(2, \psi \mathbf{N}_{K}\right)= \pm \frac{\mathfrak{f}_{p}(f, \psi)}{\mathcal{C}_{E}} \log _{E, p}\left(P_{\psi}\right) .
$$

\section{KATZ $p$-ADIC $L$-FUNCTION}

Keep the notation and assumptions of the previous section. Denote by $\Sigma_{K}$ the set of Hecke characters of $K$ of conductor dividing $\mathfrak{c}$, and define

$$
\begin{aligned}
& \Sigma_{K}^{(1)}=\left\{\psi \in \Sigma \text { of infinity type }\left(\kappa_{1}, \kappa_{2}\right), \kappa_{1} \leq 0, \kappa_{2} \geq 1\right\}, \\
& \Sigma_{K}^{(2)}=\left\{\psi \in \Sigma \text { of infinity type }\left(\kappa_{1}, \kappa_{2}\right), \kappa_{1} \geq 1, \kappa_{2} \leq 0\right\} .
\end{aligned}
$$

For all $\psi \in \Sigma_{K}^{(2)}, s=0$ is a critical point for the Hecke $L$-function $L\left(\psi^{-1}, s\right)$, and Katz's $p$-adic $L$-function is constructed by interpolating the (suitably normalized) values $L\left(\psi^{-1}, 0\right)$ as $\psi$ ranges over $\Sigma_{K}^{(2)}$.

More precisely, let $\hat{\Sigma}_{K}$ denote the completion of $\Sigma_{K}^{(2)}$ with respect to the compact open topology on the space of functions on a certain subset of $\mathbf{A}_{K}^{\times}$, as described in [BDP13, Section 5.2]. By the work of Katz [Ka76], there exists a $p$-adic analytic function

$$
\mathcal{L}_{p}(K): \hat{\Sigma}_{K} \longrightarrow \mathbb{C}_{p}
$$

which is characterized by the following interpolation property: for all $\psi \in \Sigma_{K}^{(2)}$ of infinity type $\left(\kappa_{1}, \kappa_{2}\right)$,

$$
\mathcal{L}_{p}(K)(\psi)=\mathfrak{a}_{\mathrm{K}}(\psi) \cdot \mathfrak{e}_{\mathrm{K}}(\psi) \cdot \mathfrak{f}_{\mathrm{K}}(\psi) \frac{\Omega_{p}^{\kappa_{1}-\kappa_{2}}}{\Omega^{\kappa_{1}-\kappa_{2}}} L_{\mathfrak{c}}\left(\psi^{-1}, 0\right),
$$

where the following hold:

- $L_{\mathfrak{c}}\left(\psi^{-1}, s\right)$ is Hecke's $L$-function associated with $\psi^{-1}$ with the Euler factors at primes dividing $\mathfrak{c}$ removed.

- $\Omega_{p} \in \mathbb{C}_{p}^{\times}$is a $p$-adic period attached to $K$, as defined in [BDP13, (140)].

- $\Omega \in \mathbb{C}^{\times}$is the complex period associated with $K$ as defined in equation (137) of [BDP13].

- $\mathfrak{a}_{\mathrm{K}}(\psi)=\left(\kappa_{1}-1\right) ! / \pi^{\kappa_{2}}$.

- $\mathfrak{e}_{\mathrm{K}}(\psi)=(1-\psi(\wp) / p)\left(1-\psi^{-1}(\bar{\wp})\right)$.

- $\mathfrak{f}_{\mathrm{K}}(\psi)=\left(\sqrt{D_{K}} / 2\right)^{\kappa_{2}}$. 
The following result is commonly known as Katz's Kronecker $p$-adic limit formula. It computes the value of $\mathcal{L}_{p}(K)$ at a finite-order character $\psi$ of $G_{K}$, which lies outside the region of interpolation (cf. [Ka76, Sections 10.4, 10.5]):

$$
L_{p}(K)(\psi)=\mathfrak{f}_{p}(\psi) \cdot \log _{p}\left(u_{\psi}\right),
$$

where

$$
\mathfrak{f}_{p}(\psi)= \begin{cases}\frac{1}{2}\left(\frac{1}{p}-1\right) & \text { if } \psi=1, \\ \frac{-1}{24 c}(1-\psi(\bar{\wp}))\left(1-\frac{\psi(\bar{\wp})}{p}\right) & \text { if } \psi \neq 1 .\end{cases}
$$

Here, $c>0$ is the smallest positive integer in the conductor ideal of $\psi$.

\section{Proof of Theorem 1.1}

Let

$$
f \in S_{2}\left(p N_{f}\right), \quad g=\theta_{\psi_{g}} \in M_{1}\left(D_{K} N\left(\mathfrak{c}_{g}\right), \bar{\chi}\right), \quad h=\theta_{\psi_{h}} \in M_{1}\left(D_{K} N\left(\mathfrak{c}_{h}\right), \chi\right)
$$

be the three modular forms considered in the Introduction, and we keep the as-

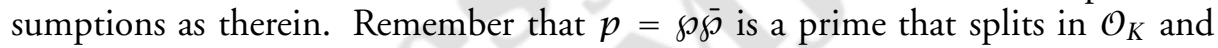
does not divide $c N_{f}$.

Let $\mathbf{f}$ and $\mathbf{g}$ be cuspidal Hida families passing through $f$ and $g_{\alpha}$, that is to say, satisfying $\mathbf{f}_{2}=f$ and $\mathbf{g}_{1}=g_{\alpha}$. The Hida family $\mathbf{f}$ is unique thanks to the classical work of Hida [Hi86]. The Hida family $\mathbf{g}$ is also unique thanks to the classicality hypothesis assumed in the Introduction, by a recent result of Bellaiche and Dimitrov [BeDi]. This family is in fact $\mathrm{CM}$, and its higher-weight classical specializations admit an explicit description that we now review.

Take a Hecke character $\lambda$ with image in $\mathbb{Z}_{p}^{\times}$of infinity type $(0,1)$ and conductor $\bar{\wp}$. Following [Hi93, pp. 235-236], with a slight modification, we define the Hecke characters $\psi_{g, \ell-1}$, of conductor $\mathfrak{c}_{\mathfrak{g}}$, as follows:

$$
\begin{aligned}
\psi_{g, \ell-1}(\mathfrak{q}) & :=\psi_{g}(\mathfrak{q})\langle\lambda\rangle^{\ell-1}(\mathfrak{q}), \quad \text { for } \mathfrak{q} \neq \bar{\wp}, \\
\psi_{g, \ell-1}(\bar{\wp}) & :=\bar{\chi}(p) \frac{p^{\ell-1}}{\psi_{\mathfrak{g}, \ell-1}(\wp)} .
\end{aligned}
$$

For every $\ell \in \mathcal{U}_{g}^{\mathrm{cl}}$, one then has that $\mathbf{g}_{\ell}$ is the ordinary $p$-stabilisation of $g_{\ell}:=\theta_{\psi_{g, \ell-1}}$. Using the notation introduced above, note that we have

$$
\alpha_{g_{\ell}}=\psi_{g, \ell-1}(\wp), \quad \beta_{g_{\ell}}=\psi_{g, \ell-1}(\bar{\wp}) .
$$

Recall the three ring class characters $\psi_{0}, \psi_{1}, \psi_{2}$ defined in the Introduction. Recall also that $\mathbf{N}_{K}$ stands for the Hecke character of infinity type $(1,1)$ induced 
by the norm from $K$ to $\mathbb{Q}$. Associated with them there are three additional families of characters which play a key role in the proof:

$$
\begin{aligned}
\Phi_{g}(\ell):= & \left(\psi_{g, \ell-1}^{2} \chi\right)^{-1} \mathbf{N}_{K}^{\ell}, \\
& \text { for which } \Phi_{g}(1)=\psi_{0}^{\prime} \mathbf{N}_{K}, \\
\Psi_{g h}(k, \ell):= & \left(\psi_{g, \ell-1} \psi_{h}\right)^{-1} \mathbf{N}_{K}^{(k+\ell-1) / 2}, \\
& \text { for which } \Psi_{g h}(2,1)=\psi_{1}^{\prime} \mathbf{N}_{K}, \\
\Psi_{g h^{\prime}}(k, \ell):= & \left(\Psi_{g, \ell-1} \psi^{\prime}{ }_{h}\right)^{-1} \mathbf{N}_{K}^{(k+\ell-1) / 2}, \\
& \text { for which } \Psi_{g h}(2,1)=\psi_{2}^{\prime} \mathbf{N}_{K} .
\end{aligned}
$$

Note that $\Phi_{g}(\ell)$ is of infinity type $\left(\kappa_{1}, \kappa_{2}\right)=(\ell,-\ell+2)$, while $\Psi_{g h}(k, \ell)$ and $\Psi_{g h^{\prime}}(k, \ell)$ are of infinity type $(\kappa+\lambda,-\lambda)$ with $\kappa=k$ and $\lambda=(\ell-k-1) / 2 \geq 0$ because $\ell \geq k+1$.

Definition 5.1. Let $L$ be a number field. A function

$$
\mathfrak{f}: \mathcal{U}_{g}^{\mathrm{cl}} \rightarrow \overline{\mathbb{Q}}
$$

is $L$-admissible if it extends to a meromorphic Iwasawa function on $\mathcal{U}_{g}$ having no pole at 1 and satisfying $\mathfrak{f}(1) \in L^{\times}$. Similarly, a function

$$
\mathfrak{f}: \mathcal{U}_{f g}^{\mathrm{cl}} \rightarrow \overline{\mathbb{Q}}
$$

is $L$-admissible if it extends to a meromorphic Iwasawa function on $\mathcal{U}_{f g}$ having no pole at $(2,1)$ and satisfying $\mathfrak{f}(2,1) \in L^{\times}$.

Set $\mathfrak{a}_{\mathrm{Pet}}(\ell)=(\ell-1) ! / \pi^{\ell}$. By [DLR, Lemma 3.7 and (53)] there exists a $\mathbb{Q}\left(\psi_{g}, \psi_{h}\right)$-admissible function $\mathfrak{f}_{\text {Pet }}$ such that

$$
\left\langle g_{\ell}^{*}, g_{\ell}^{*}\right\rangle=\mathfrak{a}_{\text {Pet }}(\ell) \cdot \mathfrak{f}_{\text {Pet }}(\ell) \cdot L\left(\Phi_{g}(\ell)^{-1}, 0\right) .
$$

Lemma 5.2. The following formulae hold true:

$$
\begin{aligned}
\Phi_{g}(\ell)(\wp) & =\frac{\beta_{g_{\ell}}^{2} \chi(p)}{p^{\ell-2}}, & \Phi_{g}(\ell)(\bar{\wp}) & =\frac{p^{\ell}}{\beta_{g_{\ell}}^{2} \chi(p)}, \\
\Psi_{g h}(k, \ell)(\bar{\wp}) & =\frac{p^{(\ell+k-1) / 2}}{\beta_{g_{\ell}} \beta_{h}}, & \Psi_{g h^{\prime}}(k, \ell)(\bar{\wp}) & =\frac{p^{(\ell+k-1) / 2}}{\beta_{g_{\ell}} \alpha_{h}} .
\end{aligned}
$$

Proof. This follows from a straightforward computation.

Lemma 5.3. The following equalities hold true:

$$
\begin{aligned}
& \mathfrak{e}_{K}\left(\Phi_{g}(\ell)\right)^{2} \cdot \mathfrak{e}_{\mathrm{GH}}(k, \ell)^{2}=\mathfrak{e}_{\mathrm{BDP}}\left(k, \Psi_{g h}(k, \ell)\right)^{2} \mathfrak{e}_{\mathrm{BDP}}\left(k, \Psi_{g h^{\prime}}(k, \ell)\right)^{2}, \\
& \mathfrak{a}_{\mathrm{GH}}(k, \ell) \cdot \mathfrak{a}_{\mathrm{K}}\left(\Phi_{g}(\ell)\right)^{2}=\mathfrak{a}_{\mathrm{BDP}}\left(\Psi_{g h}(k, \ell)\right) \cdot \mathfrak{a}_{\mathrm{BDP}}\left(\Psi_{g h^{\prime}}(k, \ell)\right) \cdot \mathfrak{a}_{\mathrm{Pet}}(\ell)^{2} .
\end{aligned}
$$


Proof. The proof again follows from a straightforward computation by using Lemma 5.2 for the first equality.

Recall the factor $\omega(f, \Psi)$ appearing in (3.1) and defined in equation (5.1.11) of [BDP13]. Recall also from the remarks around the Heegner hypothesis in the Introduction the integral ideal $\mathfrak{N}$ of $\mathcal{O}_{K}$ such that $\mathrm{N}_{K / \mathbb{Q}}(\mathfrak{N})=N_{f}$.

Lemma 5.4. The following identities hold true:

$$
\begin{aligned}
& \omega\left(f, \Psi_{g h}(k, \ell)\right)=(-1)^{(\ell-1) / 2} \frac{\Psi_{g, \ell-1}(\mathfrak{N}) \psi_{h}(\mathfrak{N})}{N_{f}^{(\ell-1) / 2}}, \\
& \omega\left(f, \Psi_{g h}(k, \ell)\right)=(-1)^{(\ell-1) / 2} \frac{\Psi_{g, \ell-1}(\mathfrak{N}) \psi_{h}^{\prime}(\mathfrak{N})}{N_{f}^{(\ell-1) / 2}} .
\end{aligned}
$$

In particular, we have

$$
\omega\left(f, \Psi_{g h}(2,1)\right)=\psi_{1}(\mathfrak{N}) \quad \text { and } \quad \omega\left(f, \Psi_{g h}(2,1)\right)=\psi_{2}(\mathfrak{N}) .
$$

Proof. We limit ourselves to compute $\omega\left(f, \Psi_{g h}(k, \ell)\right)$, as the other case is very similar. For simplicity, call $\Psi:=\Psi_{g h}(k, \ell)$ the Hecke character of infinity type $(\kappa+\lambda,-\lambda)$, where $\kappa=k$ and $\lambda=(\ell-k-1) / 2$. Define $\Psi_{\lambda}:=\Psi \mathbf{N}_{K}^{\lambda}$; then, choose an ideal $\mathfrak{b} \subset \mathcal{O}_{c}$ relatively prime to $N_{f} \mathcal{C}$ and an element $b_{N} \in \mathcal{O}_{c}$ such that $\mathfrak{b N}=\left(b_{N}\right)$. Since $\omega_{f}=1$ and $\varepsilon_{f}=1$ in our case, [BDP13, equation (5.1.11)] shows that

$$
\omega(f, \Psi)=\Psi_{\lambda}(\mathfrak{b}) \cdot(-1)^{\kappa / 2+\lambda} N^{\kappa / 2+\lambda} b_{N}^{-\kappa-2 \lambda} .
$$

By [BDP13, equation (5.1.2)], it then follows that

$$
\Psi_{\lambda}(\mathfrak{b}) \Psi_{\lambda}(\mathfrak{N})=\Psi_{\lambda}\left(\left(b_{N}\right)\right)=b_{N}^{\kappa+2 \lambda} .
$$

Substituting into (5.5), we find

$$
\omega(f, \Psi)=(-1)^{\kappa / 2+\lambda} \frac{N_{f}^{\kappa / 2+\lambda}}{\Psi_{\lambda}(\mathfrak{N})}=(-1)^{\kappa / 2+\lambda} \frac{N_{f}^{\kappa / 2}}{\Psi(\mathfrak{N})} .
$$

Using now the definition of $\Psi_{g h}(k, \ell)$ and the fact that $\kappa+2 \lambda=\ell-1$, we find

$$
\begin{aligned}
\omega(f, \Psi) & =(-1)^{(\ell-1) / 2} \cdot \frac{\psi_{g, \ell-1}(\mathfrak{N}) \psi_{h}(\mathfrak{N}) N_{f}^{k / 2}}{N_{f}^{(\ell+k-1) / 2}} \\
& =(-1)^{(\ell-1) / 2} \cdot \frac{\psi_{g, \ell-1}(\mathfrak{N}) \psi_{h}(\mathfrak{N})}{N_{f}^{(\ell-1) / 2}},
\end{aligned}
$$

as claimed. 
Let

$$
L_{0}=K\left(\psi_{g}, \psi_{h}, \tau(\chi), \sqrt{N_{g}}, \sqrt{N_{h}}\right)
$$

denote the extension of $\mathbb{Q}$ generated by the values of the Hecke characters $\psi_{g}$ and $\psi_{h}$, the Gauss sum associated with the Dirichlet character $\chi$, and the square roots of $N_{g}$ and $N_{h}$.

Fix test vectors $\breve{\mathbf{f}}, \breve{\mathbf{g}}$, and $\breve{h}$ as in Section 2 , giving rise to the $p$-adic $L$-function $\mathcal{L}_{p}^{g}(\breve{\mathbf{f}}, \breve{\mathbf{g}}, \breve{h})$ satisfying the good properties discussed around (2.2).

Theorem 5.5. There exist a quadratic extension $L / L_{0}$ and an $L$-admissible function $\mathfrak{f}$ on $\mathcal{U}_{f g}$ such that the following factorization of two-variable $p$-adic L-functions holds:

$$
\begin{aligned}
\mathcal{L}_{p}^{\mathcal{g}}(\breve{\mathbf{f}}, \breve{\mathbf{g}}, \breve{h})(k, \ell) \cdot \mathcal{L}_{p}(K)\left(\Psi_{\mathbf{g}}(\ell)\right) \\
\quad=\mathfrak{f}(k, \ell) \cdot \mathcal{L}_{p}(\mathbf{f} / K)\left(k, \Psi_{\mathbf{g} h}(k, \ell)\right) \cdot \mathcal{L}_{p}(\mathbf{f} / K)\left(k, \Psi_{\mathbf{g} h^{\prime}}(k, \ell)\right) .
\end{aligned}
$$

Proof. We have a decomposition of classical $L$-functions given by

$$
\begin{aligned}
L\left(f_{k}\right. & \left.\otimes g_{\ell} \otimes h, \frac{k+\ell-1}{2}\right) \\
& =\mathcal{E} u l_{N}(k, \ell) \cdot L\left(f_{k}, \Psi_{g h}(k, \ell)^{-1}, 0\right) \cdot L\left(f_{k}, \Psi_{g h^{\prime}}(k, \ell)^{-1}, 0\right) .
\end{aligned}
$$

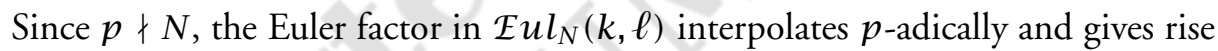
to a two-variable $L_{0}$-admissible function. Combine equations (5.4) and (5.6) with the interpolation formula (2.1). Then, use equations (3.1) and (4.1) to replace the classical $L$-functions with their respective $p$-adic avatar. An easy check shows that the periods simplify.

Define

$$
\omega(k, \ell):=I\left(\breve{g}_{\ell}^{*}, \breve{f}_{k}^{*}, h^{*}\right) / I\left(\breve{g}_{\ell}, \breve{f}_{k}, \breve{h}\right)
$$

as in [DLR, (25)]. Recall also the local constants $W_{v}$ for $v \mid N \infty$ introduced in (2.2).

By Lemma 5.3 one obtains the following equality, true for every $(k, \ell) \in \mathcal{U}_{f g}^{\mathrm{cl}}$ :

$$
\begin{aligned}
\mathcal{L}_{p}^{\mathcal{g}}(\breve{\mathbf{f}}, \breve{\mathbf{g}}, \breve{h})(k, \ell)^{2} \cdot \mathcal{L}_{p}(K)\left(\Phi_{g}(\ell)\right)^{2} \\
\quad=\mathfrak{f}_{0}(k, \ell) \cdot \mathcal{L}_{p}(\mathbf{f} / K)\left(k, \Psi_{g h}(k, \ell)\right)^{2} \cdot \mathcal{L}_{p}(\mathbf{f} / K)\left(k, \Psi_{g h^{\prime}}(k, \ell)\right)^{2}
\end{aligned}
$$

where

$$
\mathfrak{f}_{0}(k, \ell)=\frac{\mathcal{E} u l_{N}(k, \ell)}{\mathfrak{f}_{\mathrm{Pet}}(\ell)^{2}} \cdot \frac{\mathfrak{f}_{\mathrm{GH}}(\ell, k, 1) \cdot \mathfrak{f}_{\mathrm{K}}\left(\Phi_{g}(\ell)\right)^{2}}{\mathfrak{f}_{\mathrm{BDP}}\left(\Psi_{g h}(k, \ell)\right) \cdot \mathfrak{f}_{\mathrm{BDP}}\left(\Psi_{g h^{\prime}}(k, \ell)\right)} \cdot \frac{\prod_{v \mid N \infty} W_{v}}{\omega(k, \ell)} .
$$

Let us show that $\mathfrak{f}_{0}$ is $L_{0}$-admissible. First of all, it is immediate to verify that the powers of $\pi$ appearing in the various fudge factors cancel out. Notice also that 
almost all terms appearing in the numerator and denominator of the definition of $\mathfrak{f}_{0}(k, \ell)$ are $L_{0}$-admissible for obvious reasons, following directly from the definitions. This is the case except for the constants $W_{v}, \omega\left(f_{k}, \Psi_{g h}\right) \omega\left(f_{k}, \Psi_{g h^{\prime}}\right)$, and $\omega(k, \ell)$, which need to be considered in more detail.

As for the local constants $W_{v}$, we already argued in Section 2 that for $v \mid N$ the test vectors can be chosen in such a way that $W_{v}$ lie in $\mathbb{Q}^{\times}$and do not depend on $(k, \ell)$. Moreover, the recent results of [Wo2] guarantee that in this setting $W_{\infty}=1$ for all $(k, \ell)$.

As for the global constant $\omega\left(f_{k}, \Psi_{g h}\right) \omega\left(f_{k}, \Psi_{g h^{\prime}}\right)$, using Lemma 5.4 one derives that

$$
\omega\left(f_{k}, \Psi_{g h}\right) \omega\left(f_{k}, \Psi_{g h^{\prime}}\right)=\frac{\psi_{g, \ell-1}(\mathfrak{N})^{2} \psi_{h}(\mathfrak{N}) \psi_{h}^{\prime}(\mathfrak{N})}{N_{f}^{\ell-1}} .
$$

Since $p \nmid N_{f}$, it follows that the function $\omega\left(f, \Psi_{g h}\right) \omega\left(f, \Psi_{g h^{\prime}}\right)$ is $L_{0}$-admissible (in fact, it is $K\left(\psi_{g}, \psi_{h}\right)$-admissible).

The $L_{0}$-admissibility of the function $\omega(k, \ell)$ follows by the same argument as in the last part of the proof of [DLR, Theorem 3.9]. Hence, we have proved that $\mathfrak{f}_{0}$ is $L_{0}$-admissible, and the theorem follows after taking the square roots on both sides of (5.7). that

Corollary 5.6 (Elliptic Stark conjecture). There exists a scalar $\lambda \in L^{\times}$such

$$
\int_{\breve{\gamma}_{g_{\alpha}}} \breve{f} \cdot \breve{g}=\lambda \cdot \frac{\log _{E, p}\left(P_{\psi_{1}^{\prime}}\right) \log _{E, p}\left(P_{\psi_{2}^{\prime}}\right)}{\log _{p}\left(u_{\psi_{0}^{\prime}}\right)} .
$$

Proof. Notice that we have

$$
\Phi_{g}(1)=\psi_{g}^{\prime} / \psi_{g}=\psi_{0}^{\prime}, \quad \Psi_{g h}(2,1)=\psi_{1}^{\prime} \quad \text { and } \quad \Psi_{g h^{\prime}}(2,1)=\psi_{2}^{\prime} .
$$

Using Theorem 5.5 and equations (2.3), (3.2), and (4.2), one obtains the desired result.

\section{AN EXPLICIT FORMULA}

Let us assume now that $\psi=\psi_{g}$ is a ring class character of conductor $c \in \mathbb{Z}_{\geq 1}$, and $\psi_{h}=1$ is the trivial character. This implies that $h=\theta_{\psi_{h}}=E_{1, \chi_{K}}$ is the Eisenstein series associated with the pair of Dirichlet characters $\left(1, \chi_{K}\right)$. In this setting, the characters defined in equations (5.2) and (5.3) coincide, that is, $\Psi_{g h}=\Psi_{g h^{\prime}}$, and thus we simply denote this character $\Psi_{g}$.

In this section, we exploit the results obtained previously to prove an explicit formula for the scalar $\lambda$ that makes its appearance in Theorem 1.1, in the particular scenario considered here. In order to do so we invoke yet another $p$-adic $L$-function in addition to the ones introduced so far: namely, the Hida-Rankin 
$p$-adic $L$-function associated by Hida in Chapter 10 of [Hi93] to the convolution of two Hida families $\mathbf{f}, \mathbf{g}$ of cusp forms.

We follow the conventions and notation employed in [BDR1, Section 2] and [CR, Section 3], where Hida-Rankin's $p$-adic $L$-function $\mathcal{L}_{p}^{\mathcal{g}}(\mathbf{f}, \mathbf{g})$ is described as a function on $\mathcal{U}_{f g}$ and is characterized by the following interpolation property: for every $(k, \ell) \in \mathcal{U}_{f g}^{\mathrm{cl}}$ such that $\ell \geq k+1$, it holds that

$$
\mathcal{L}_{p}^{g}(\mathbf{f}, \mathbf{g})(k, \ell)=\mathfrak{e}_{\mathrm{HR}}(k, \ell) \cdot \mathfrak{a}_{\mathrm{HR}}(k, \ell) \cdot \mathfrak{f}_{\mathrm{HR}}(k, \ell) \cdot \frac{L\left(\mathbf{f}_{k} \otimes \mathbf{g}_{\ell},(\ell+k-1) / 2\right)}{\left\langle\mathbf{g}_{\ell}, \mathbf{g}_{\ell}\right\rangle_{N}},
$$

where

- $\mathfrak{e}_{\mathrm{HR}}(k, \ell)=\mathfrak{e}_{\mathrm{GH}}(k, \ell, 1)$.

- $\mathfrak{a}_{\mathrm{HR}}(k, \ell)=N / \sqrt{D_{K}}$.

- $\mathfrak{f}_{\mathrm{HR}}(k, \ell)=(-1)^{(\ell-k-1) / 2} \cdot((\ell-k-1) / 2) !((\ell-k-3) / 2) ! \cdot 2^{-2 \ell+1} \pi^{-\ell}$.

Moreover, by [CR, Proposition 3.2] we dispose of the following formula for the value of $\mathcal{L}_{p}^{g}(\mathbf{f}, \mathbf{g})$ at the point $(k, \ell)=(2,1)$ lying outside the region of interpolation. Keeping the notation of the Introduction and Section 2, we have

$$
\mathcal{L}_{p}^{g}(\mathbf{f}, \mathbf{g})(2,1)=\int_{\breve{\gamma} g_{\alpha}} \breve{f} \cdot \breve{h} .
$$

Recall the number field appearing in the statement of Theorem 5.5.

Proposition 6.1. There exists a two-variable L-admissible function $\mathfrak{f}(k, \ell)$ such that the following factorization of $p$-adic L-functions holds:

$$
\mathcal{L}_{p}^{g}(\mathbf{f}, \mathbf{g})(k, \ell) \cdot \mathcal{L}_{p}(K)\left(\Phi_{g}(\ell)\right)=\mathfrak{f}(k, \ell) \cdot \mathcal{L}_{p}(\mathbf{f} / K)\left(\Psi_{g h}(k, \ell)\right)^{2} .
$$

Proof. After setting $\Psi:=\Psi_{g h}(k, \ell)$, it is easy to verify that

$$
L\left(\breve{g}_{\ell} \otimes \breve{f}_{k},(\ell+k-1) / 2\right)=\mathcal{E} u l_{N}(k, \ell) \cdot L\left(f_{k}, \Psi, 0\right)
$$

where $\mathcal{E} u l_{N}(k, \ell)$ stands for a product of Euler factors at primes dividing $N$. The

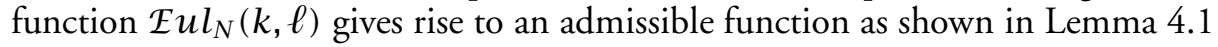
of [CR]. From this, the proof proceeds along lines very similar to those in the proof of Theorem 5.5 and [CR, Theorem 4.4], if we replace the interpolation formula of the Garrett-Hida triple-product $L$-function by the one provided above.

We are content here to record an explicit expression for the admissible function appearing in the above statement, namely,

$$
\begin{aligned}
\mathfrak{f}(k, \ell)= & \mathcal{E} u l_{N}(k, \ell) \cdot(-1)^{k / 2} \\
& \times \frac{\operatorname{Im}\left(D_{K} c^{2}\right)}{\operatorname{Im}(N)} \frac{N \cdot 2^{-\#\left\{q \mid\left(D_{K}, N_{f}\right)\right\}}}{h_{c} \cdot D_{K}} \frac{\psi_{\ell-1}(\mathfrak{N})}{c^{\ell+1} N_{f}^{(\ell-1) / 2}},
\end{aligned}
$$


where for any integer $M \geq 1$ we set

$$
\operatorname{Im}(M):=\left[\mathrm{SL}_{2}(\mathbb{Z}): \Gamma_{0}(M)\right]=\prod_{q^{n_{q} \| M}} q^{n_{q}-1}(q+1) .
$$

Corollary 6.2. Assume $N_{f}=D_{K}$ and $g=h=\operatorname{Eis}_{1}\left(1, \chi_{K}\right)$. Then,

$$
\int_{\gamma_{g \alpha}} f \cdot h=\lambda \cdot \frac{\log _{E, p}^{2}\left(P_{\psi^{\prime}}\right)}{\log _{p}\left(u_{\psi^{\prime 2}}\right)}
$$

with

$$
\lambda=\frac{\left(p-a_{p}(f) \psi(\wp)\right)^{2}}{p \cdot c_{E}^{2}} \cdot \frac{\lambda_{0}}{h_{K} g_{K}},
$$

where

$$
\lambda_{0}= \begin{cases}\frac{1}{p-1} & \text { if } \psi^{2}=1, \\ \frac{12}{p-(p+1) \psi^{2}(\bar{\wp})+\psi^{4}(\bar{\wp})} & \text { that is to say, if } g \text { is an Eisenstein series, } \\ & \text { that is to say, if } g \text { is cuspidal. }\end{cases}
$$

Proof. The value of the admissible function $\mathfrak{f}$ appearing in (6.2) at the point $(k, \ell)=(2,1)$ is

$$
\mathfrak{f}(2,1)=-\frac{2 \psi(\mathfrak{N})}{h_{K} g_{K}} .
$$

The result now follows upon combining this with formulae (3.2), (4.2), and (6.1).

Acknowledgements. The two authors were supported by Spanish Grant MTM2015-63829-P. The first author acknowledges financial support by the Spanish Ministry of Economy and Competitiveness, through the Severo Ochoa programme for Centres of Excellence in R\&D (SEV-2015-0554). The second author acknowledges financial support from the Spanish Ministry of Economy and Competitiveness, through the María de Maeztu Programme for Units of Excellence in R\&D (MDM-2014-0445). This project has also received funding from the European Research Council (ERC) under the European Union's Horizon 2020 research and innovation programme (grant agreement no. 682152). Finally, the authors acknowledge financial support from the Spanish Ministry of Economy and Competitiveness, through the Maria de Maeztu Programme for Units of Excellence in R\&D (MDM-2014-0445). 


\section{REFERENCES}

[ARS] A. Agashe, K. Ribet, And W.A. Stein, The Manin constant, Pure Appl. Math. Q. 2 (2006), no. 2, Special Issue: In honor of John H. Coates, 617-636. http://dx.doi.org/ 10.4310/PAMQ.2006.v2.n2.a11. MR2251484.

[BeDi] J. BellaïCHE AND M. Dimitrov, On the eigencurve at classical weight 1 points, Duke Math. J. 165 (2016), no. 2, 245-266, available at http://math.univ-lille1.fr/ $\sim$ mladen/. http://dx.doi.org/10.1215/00127094-3165755. MR3457673.

[BD1] M. BERTOLINI AND H. DARMON, Kato's Euler system and rational points on elliptic curves I: A p-adic Beilinson formula, Israel J. Math. 199 (2014), no. 1, 163-188. http://dx.doi. org/10.1007/s11856-013-0047-2. MR3219532.

[BD3] - The rationality of Stark-Heegner points over genus fields of real quadratic fields, Ann. of Math. (2) 170 (2009), no. 1, 343-370. http://dx.doi.org/10.4007/annals. 2009. 170.343. MR2521118.

[BDR1] M. Bertolini, H. Darmon, And V. Rotger, Beilinson-Flach elements and Euler systems I: Syntomic regulators and p-adic Rankin L-series, J. Algebraic Geom. 24 (2015), no. 2, 355-378. http://dx.doi.org/10.1090/S1056-3911-2014-00670-6. MR3311587.

[BDP13] M. Bertolini, H. Darmon, And K. PRASAnna, Generalized Heegner cycles and padic Rankin L-series, Duke Math. J. 162 (2013), no. 6, 1033-1148, With an appendix by Brian Conrad. http://dx.doi.org/10.1215/00127094-2142056. MR3053566.

[Ca1] F. CASTELlÀ, On the p-adic variation of Heegner points, Journal de l'Inst. Math. de Jussieu, to appear.

[Ca2] On the exceptional specializations of big Heegner points, J. Inst. Math. Jussieu 17 (2018), no. 1, 207-240. http://dx.doi.org/10.1017/S1474748015000444. MR3742560.

[CR] D. CasazZa And V. ROTGER, Stark points and p-adic iterated integrals, Ramanujan Journal of Math., to appear.

[Dar] H. DARMON, Integration on $\mathcal{H}_{p} \times \mathcal{H}$ and arithmetic applications, Ann. of Math. (2) 154 (2001), no. 3, 589-639. http://dx.doi.org/10.2307/3062142. MR1884617.

[DLR] H. DARMON, A. LAUDER, AND V. ROTGER, Stark points and p-adic iterated integrals attached to modular forms of weight one, Forum Math. Pi 3 (2015), e8, 95. http://dx. doi. org/10.1017/fmp.2015.7. MR3456180.

[DR1] H. DARMON AND V. ROTGER, Diagonal cycles and Euler systems I: A p-adic Gross-Zagier formula, Ann. Sci. Éc. Norm. Supér. (4) 47 (2014), no. 4, 779-832 (English, with English and French summaries). http://dx.doi.org/10.24033/asens.2227. MR3250064.

[DR2] Diagonal cycles and Euler systems II: The Birch and Swinnerton-Dyer conjecture for Hasse-Weil-Artin L-functions, J. Amer. Math. Soc. 30 (2017), no. 3, 601-672. http://dx. doi.org/10.1090/jams/861. MR3630084.

[DR4] A Kolyvagin theorem in rank 2, in progress.

[DG] M. Dimitrov And E. GHATE, On classical weight one forms in Hida families, J. Théor. Nombres Bordeaux 24 (2012), no. 3, 669-690 (English, with English and French summaries). MR3010634.

[Gar] P.B. GARRETT, Decomposition of Eisenstein series: Rankin triple products, Ann. of Math. (2) 125 (1987), no. 2, 209-235. http://dx.doi.org/10.2307/1971310. MR881269.

[Gro] B.H. GROSs, Heegner points on $X_{0}(N)$, Heegner points and Rankin L-series, Cambridge University Press (Mathematical Sciences Research Institute Publications), 2004, pp. Chapter 5. http://dx. doi.org/10.1017/CB09780511756375.

[Hi86] H. HIDA, Galois representations into $\mathrm{GL}_{2}\left(\mathbb{Z}_{p} \llbracket X \rrbracket\right)$ attached to ordinary cusp forms, Invent. Math. 85 (1986), no. 3, 545-613. http://dx.doi.org/10.1007/BF01390329. MR848685.

[Hi93] _ Elementary Theory of L-functions and Eisenstein Series, London Mathematical Society Student Texts, vol. 26, Cambridge University Press, Cambridge, 1993. http://dx. doi.org/10.1017/CB09780511623691. MR1216135. 
[Ho07] B. HowARD, Variation of Heegner points in Hida families, Invent. Math. 167 (2007), no. 1, 91-128. http://dx.doi.org/10.1007/s00222-006-0007-0. MR2264805.

[Ja88] U. Jannsen, Continuous étale cohomology, Math. Ann. 280 (1988), no. 2, 207-245. http://dx.doi.org/10.1007/BF01456052. MR929536.

[Ka10] E. KANI, Binary theta series and modular forms with complex multiplication, Int. J. Number Theory 10 (2014), no. 4, 1025-1042. http://dx.doi.org/10.1142/ S1793042114500134. MR3208873.

[Ka76] N.M. KATZ, p-adic interpolation of real analytic Eisenstein series, Ann. of Math. (2) 104 (1976), no. 3, 459-571. http://dx.doi.org/10.2307/1970966. MR0506271.

[Ka78] _ p-adic L-functions for CM fields, Invent. Math. 49 (1978), no. 3, 199-297. http://dx.doi.org/10.1007/BF01390187. MR513095.

[KZ84] W. KOHNEn AND D. ZAGIER, Modular forms with rational periods, Modular Forms, Durham (1983), Ellis Horwood Ser. Math. Appl.: Statist. Oper. Res., Horwood, Chichester, 1984, pp. 197-249. MR803368.

[LRdV] M. Longo, V. Rotger, AND C. De Vera-Piquero, Heegner points on HijikataPizer-Shemanske curves and the Birch and Swinnerton-Dyer conjecture, Publ. Mat. 62 (2018), no. 2, 355-396. http://dx.doi.org/10.5565/PUBLMAT6221803. MR3815284.

[PR93] B. PERRIN-RIOU, Fonctions L p-adiques d'une courbe elliptique et points rationnels, Ann. Inst. Fourier (Grenoble) 43 (1993), no. 4, 945-995 (French, with English and French summaries). MR1252935.

[Pr90] D. PRASAD, Trilinear forms for representations of GL(2) and local $\varepsilon$-factors, Compositio Math. 75 (1990), no. 1, 1-46. MR1059954.

[Wi88] A. WILES, On ordinary $\lambda$-adic representations associated to modular forms, Invent. Math. 94 (1988), no. 3, 529-573. http://dx.doi.org/10.1007/BF01394275. MR969243.

[Wo1] M. WOODBURY, Trilinear forms and subconvexity of the triple product L-function, submitted.

[Wo2] _ On the triple product formula: Real local calculations, submitted.

DANIELE CASAZZA:

ICMAT - CSIC

C. de Nicolás Cabrera 13-15

28049 Madrid, Spain

E-MAIL: daniele.casazza@icmat.es

VICTOR ROTGER:

Departament de Matemàtica Aplicada II

Universitat Politècnica de Catalunya

C. Jordi Girona 1-3

08034 Barcelona, Spain

E-MAIL: victor.rotger@upc.edu

Received: May 31, 2017. 